\title{
Protective effect of dimethyl fumarate on oxidative damage and signaling in cardiomyocytes
}

\author{
YUANYUAN KUANG ${ }^{1}$, YINZHUANG ZHANG ${ }^{2}$, ZHEN XIAO $^{1}$, LIJUN XU $^{1}$, PING WANG $^{1}$ and QILIN MA $^{1}$ \\ ${ }^{1}$ Department of Cardiovascular Medicine, Xiangya Hospital, Central South University, Changsha, Hunan 410008; \\ ${ }^{2}$ Department of Cardiovascular Medicine, The First Hospital of Changsha, Changsha, Hunan 410005, P.R. China
}

Received March 1, 2020; Accepted June 26, 2020

DOI: $10.3892 / \mathrm{mmr} .2020 .11342$

\begin{abstract}
Myocardial ischemia/reperfusion (I/R) injury contributes to the pathogenesis of numerous diseases. Based on its antioxidant and anti-inflammatory effects, dimethyl fumarate (DMF) has been reported to exert protective effects against I/R. However, to the best of our knowledge, its potential role as a myocardial protective agent in heart disease has received little attention. Previous studies have suggested that DMF may exert its protective effects by activating nuclear factor erythroid 2-related factor 2 (Nrf2); however, the exact underlying mechanisms remain to be elucidated. The aim of the present study was to investigate the protective role of DMF in myocardial $\mathrm{I} / \mathrm{R}$ injury, and to determine the role of $\mathrm{Nrf} 2$ in mediating the activity of DMF. H9c2 cells were incubated with DMF $(20 \mu \mathrm{M})$ for $24 \mathrm{~h}$ before establishing the I/R model, and were then subjected to myocardial ischemia for $6 \mathrm{~h}$, followed by reperfusion. Cell viability, lactate dehydrogenase levels, anti-oxidant enzyme expression levels and anti-apoptotic effects were evaluated, and AKT/Nrf2 pathway-associated mechanisms were investigated. The results of the present study indicated that DMF may reduce myocardial I/R injury in a Nrf2-dependent manner. DMF significantly improved cellular viability, suppressed the expression of apoptotic markers, decreased the production of reactive oxygen species and increased the expression of Nrf2-regulated antioxidative genes. Notably, these beneficial DMF-mediated effects were not observed in the control or I/R groups. In conclusion, the results of the present study suggested that DMF may exert protective effects against a myocardial I/R model, and further validated Nrf2 modulation as a primary mode of action. Thus suggesting that DMF may be a potential therapeutic agent for AKT/Nrf2 pathway activation in myocardial, and potentially systemic, diseases.
\end{abstract}

Correspondence to: Professor Qilin Ma, Department of Cardiovascular Medicine, Xiangya Hospital, Central South University, 87 Xiangya Road, Changsha, Hunan 410008, P.R. China E-mail: mqilin2004@163.com

Key words: ischemia/reperfusion, dimethyl fumarate, oxidative stress, apoptosis

\section{Introduction}

Ischemic heart disease remains the leading cause of death and is a critical threat to public health worldwide (1). Considerable advancements have been made in the acute care of patients with ischemic heart disease, particularly timely reperfusion interventions, through either percutaneous coronary intervention (PCI) or coronary artery bypass graft surgery (CABG) (2). However, the clinical outcome of these therapeutic developments remains unsatisfactory (3), which is largely due to myocardial damage, including ischemia/reperfusion (I/R) injury brought about by timely reperfusion treatment (4).

Myocardial I/R is known to result in myocardial damage, including necrosis and/or tissue degradation, heart failure, myocardial stunning, no-reflow and reperfusion arrhythmia (5). These factors have a significant impact on the clinical outcomes of patients with ischemic heart disease, including myocardial infarct size and dysfunction (6). Although the underlying mechanisms of I/R-induced myocardial injury are not completely understood (7), various studies have reported that a series of pathophysiological alterations are involved in this process (8). Among these, cellular hypoxia/reoxygenation may simulate a state of I/R (9), where cellular oxidative stress serves an important role in the activation of diverse pathways resulting in myocardial injury (10). Oxidative stress has been reported to impair mitochondrial function (11) and promote the excessive production of reactive oxygen species (ROS) (12). This results in an imbalance between ROS production and radical scavenging, and thus a disturbed redox status (13). It is evident that this altered redox status may activate a series of cellular processes, including apoptosis, which is reported to be critically involved in I/R-associated myocardial injury (14).

In this context, in order to improve the clinical outcome of ischemic heart disease, measures that counteract the cellular mechanisms of I/R-induced myocardial damage are of great importance. In previous decades, effective measures for protecting the myocardium from I/R injury have been investigated (5). Pre- and post-conditioning with physical or pharmacological strategies have been extensively researched (15), and although the results of experimental studies appear promising, those of clinical trials have been disappointing (16). Therefore, the investigation of effective treatments to protect against myocardial I/R injury are of considerable scientific and clinical significance. 
Dimethyl fumarate (DMF) is derived from fumarate acid esters and has historically been used to treat patients with relapsing-remitting multiple sclerosis (17). In addition, DMF has been reported to exert protective effects against $\mathrm{I} / \mathrm{R}$ injury in the brain (18). DMF is recognized as a potent antioxidant (19) that exerts profound effects on the modulation of apoptosis (20). It has also been shown to activate the nuclear factor erythroid 2-related factor 2 (Nrf2) signaling pathway. Nrf2 is located in the cytoplasm and is bound to the cytosolic protein Kelch-like ECH-associated protein 1 (Keap1), which subsequently regulates its activity. The Nrf2/Keap1 complex is rapidly recycled via a series of ubiquitination and proteasomal degradation events, and reactive sulfhydryls that act on Keap1 are readily oxidized by ROS and electrophiles, thereby releasing Nrf2, and allowing it to translocate into the nucleus. Here, Nrf2 activates target genes that possess antioxidant response elements (AREs) in their promoter regions (21), and that regulate downstream mitochondria-mediated apoptosis via caspase-3 (22). Therefore, DMF may exert protective effects against myocardial I/R injury. However, to the best of our knowledge, this has not previously been reported. The present study aimed to investigate the potential protective effects of DMF on I/R-induced oxidative damage and signaling in a cardiomyocyte culture setting.

\section{Materials and methods}

Experimental protocol. The experimental protocol is depicted in Fig. 1. H9c2 cells were cultured to $70 \%$ confluence and grouped according to the associated treatment conditions. At the indicated time points, the media and culture conditions were changed accordingly.

Cell culture and treatments. Rat H9c2 cardiomyoblast cells were purchased from the American Type Culture Collection and cultured in Dulbecco's modified Eagle's medium (DMEM) supplemented with $10 \%$ fetal bovine serum and penicillin/streptomycin $(100 \mathrm{U} / \mathrm{ml} / 100 \mathrm{mg} / \mathrm{ml}$; all $\mathrm{NCM}$ Biotech) at $37^{\circ} \mathrm{C}\left(5 \% \mathrm{CO}_{2}\right)$ in a humidified incubator. Firstly, the rat $\mathrm{H} 9 \mathrm{C} 2$ cardiomyocytes were randomly divided into 5 groups: i) Control group; ii) $5 \mu \mathrm{M}$ DMF; iii) $10 \mu \mathrm{M}$ DMF; iv) $20 \mu \mathrm{M}$ DMF; v) $40 \mu \mathrm{M}$ DMF. Cell Counting Kit-8 (CCK-8; BioTool Service $\mathrm{GmbH}$ ) was used determine the ideal concentration of DMF that was not toxic to cells, it was found that the safest concentration of DMF was $20 \mu \mathrm{M}$. Secondly, at 70\% confluence, the cells were categorized into different groups ( $\mathrm{n}=3$ wells/group) according to treatment. The control group cells were cultured for a further $34 \mathrm{~h}$; the I/R group cells were cultured for a further $24 \mathrm{~h}$ and were then transferred to glucose-free DMEM and cultured in an anaerobic chamber $\left(95 \% \mathrm{~N} 2,5 \% \mathrm{CO}_{2}\right)$ for $6 \mathrm{~h}$, followed by $4 \mathrm{~h}$ culture under the same conditions as the control group; the DMF group was treated in the same way as the $\mathrm{I} / \mathrm{R}$ group with the exception of DMF $(20 \mu \mathrm{M})$ pretreatment (Sigma-Aldrich; Merck KGaA) for $24 \mathrm{~h}$. At the end of the culture period, the cells and media were harvested for experimentation.

Cell viability. Cell viability was determined using the CCK-8 (BioTool Service $\mathrm{GmbH}$ ). The cultured cells were washed three times with PBS and $10 \mu$ l CCK- 8 solution was added to each well prior to incubation for $1 \mathrm{~h}$ at $37^{\circ} \mathrm{C}$. Absorbance was measured at $450 \mathrm{~nm}$ using a microplate reader (Tecan Group, Ltd.). Absorbance values were recorded relative to the control group, which was set at $100 \%$.

Lactate dehydrogenase $(\mathrm{LDH})$ release. The harvested cell culture media were collected and LDH levels were quantified using a LDH colorimetric assay kit (Beyotime Institute of Biotechnology), according to the manufacturer's protocol. Briefly, $120 \mu \mathrm{l}$ cell culture medium was mixed with $60 \mu \mathrm{l}$ $\mathrm{LDH}$ working solution and incubated at $25^{\circ} \mathrm{C}$ for $30 \mathrm{~min}$. The absorbance at $490 \mathrm{~nm}$ was determined using a microplate reader (as aforementioned).

ROS production. ROS production was determined using a ROS Assay kit (Beyotime Institute of Biotechnology), according to the manufacturer's protocol. DCFH-DA was diluted in serum-free medium $(1: 1,000)$ to a working concentration of $10 \mu \mathrm{M}$. The cells $\left(1 \times 10^{6}\right)$ were incubated at $37^{\circ} \mathrm{C}$ for $20 \mathrm{~min}$, and then washed three times with serum-free cell culture medium to remove excess DCFH-DA. The fluorescence intensity of the stained cells was observed using an inverted fluorescence microscope (magnification, x200).

Flow cytometric analysis. Cell apoptotic rates were detected by flow cytometry using an Annexin V/propidium iodide (PI) apoptosis detection kit (Hanteng Biology). Annexin V is a sensitive indicator of early apoptosis (23), and PI is a nucleic acid-binding dye that detects late apoptotic and dead cells (23). Samples $\left(1 \times 10^{4}\right)$ were double-stained with Annexin V (2 $\left.\mu \mathrm{l}\right)$ and PI ( $5 \mu \mathrm{l})$ for $15 \mathrm{~min}$ at $25^{\circ} \mathrm{C}$ in the dark, and then evaluated using a flow cytometer (BD FACSCanto II; Becton, Dickinson and Company) and FlowJo v10.4 software (FlowJo, LLC). Through flow cytometry, cellular vital conditions could be assessed, and cells were differentiated into dead, late apoptotic, early apoptotic and living cells (24).

TdT-mediated dUTP-biotin nick end labeling (TUNEL) analysis. Cellular apoptosis was also determined at the nuclear level using the TUNEL method. TUNEL staining was performed using an In Situ Cell Death Detection kit (Roche Diagnostics), according to the manufacturer's protocol. TUNEL-positive cells were detected under an inverted fluorescence microscope and analyzed using ImageJ Software 1.8.0 (National Institutes of Health), and the optical staining values were calculated.

Western blot analysis. The harvested cells were homogenized in ice-cold lysis buffer (SDS-PAGE Sample Loading buffer; Beyotime Institute of Biotechnology) for protein extraction. Sample protein concentrations were determined using a BCA Protein Assay kit (Beyotime Institute of Biotechnology). Proteins $(20 \mu \mathrm{g})$ were loaded and separated by SDS-PAGE (10 and $12 \%$ gels), and were then transferred onto PVDF membranes, which were blocked with 4\% BSA (Sigma-Aldrich; Merck $\mathrm{KGaA}$ ) at $25^{\circ} \mathrm{C}$ and $1 \mathrm{~h}$. Membranes were then incubated at $4^{\circ} \mathrm{C}$ for $12 \mathrm{~h}$ with primary antibodies against the following proteins: GAPDH (1:1,000; cat. no. GB12002; Wuhan Servicebio Technology Co., Ltd.), cleaved caspase-3 (1:500; cat. no. 9661T; Cell Signaling Technology, Inc.), B-cell 


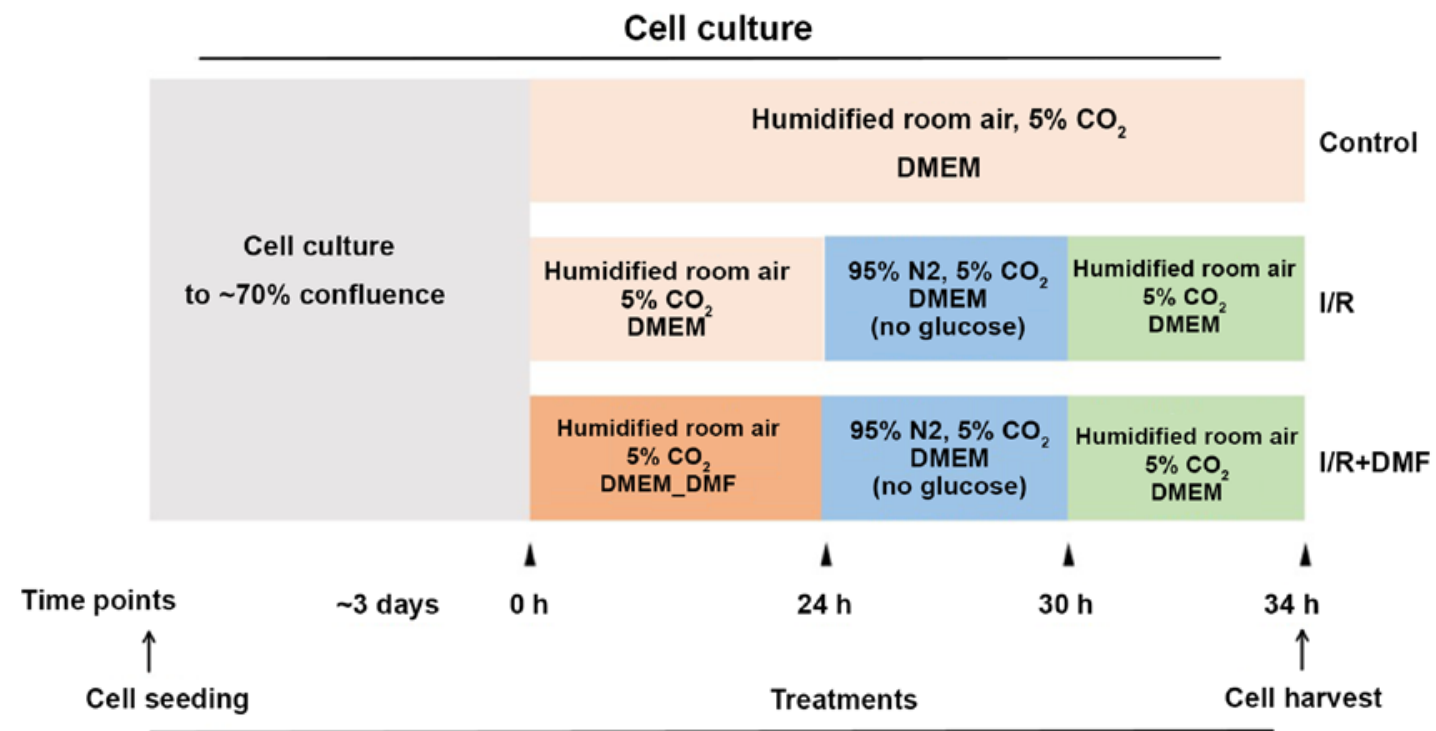

Procedure

Figure 1. Experimental protocol. H9c2 cells were seeded in DMEM and were allowed to reach $70 \%$ confluence for $\sim 3$ days before being separated into the following groups: Control, I/R and I/R + DMF. DMEM, Dulbecco's modified Eagle's medium; I/R, ischemia/reperfusion; DMF, dimethyl fumarate.
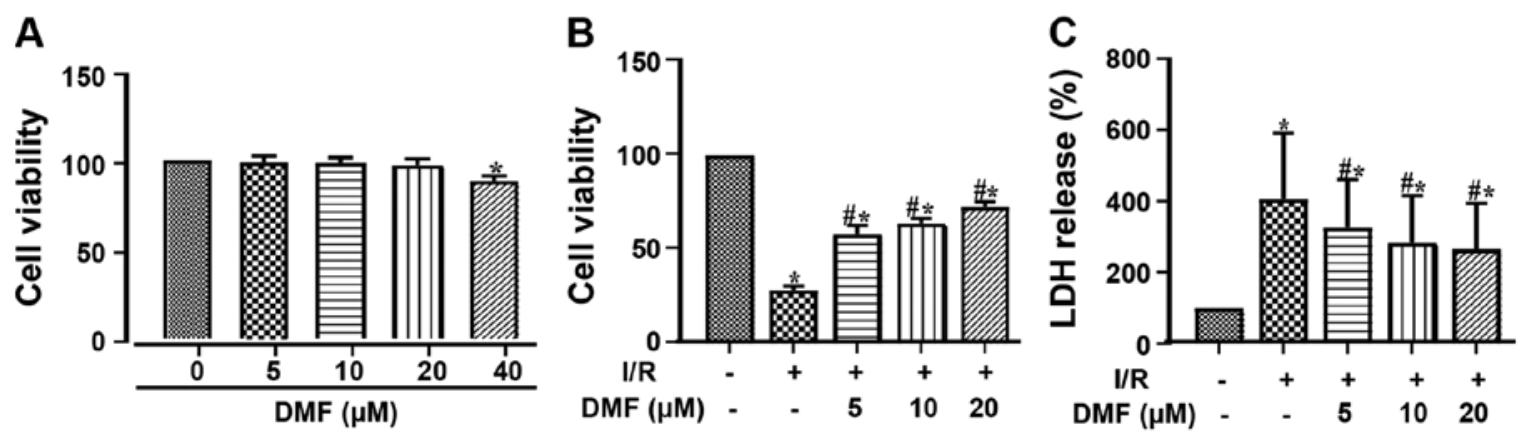

Figure 2. Effects of DMF on cell viability and damage. (A) Assessment of different doses of DMF without I/R on cell viability. Effects of I/R and DMF on (B) cell viability and (C) $\mathrm{LDH}$ release. ${ }^{*} \mathrm{P}<0.05$ vs. the control group; ${ }^{*} \mathrm{P}<0.05$ vs. the $\mathrm{I} / \mathrm{R}$ group $(\mathrm{n}=3)$. I/R, ischemia/reperfusion; DMF, dimethyl fumarate; $\mathrm{LDH}$, lactate dehydrogenase.

lymphoma 2 (Bcl-2; 1:500; cat. no. GTX100064; Genetex, Inc.), Bcl-2-associated X protein (Bax; 1:500; cat. no. 2772T; Cell Signaling Technology, Inc.), AKT (1:1,000; cat. no. 4691T; Cell Signaling Technology, Inc.), phosphorylated (p)-AKT (1:1,000; cat. no. 4060T; Cell Signaling Technology, Inc.), Nrf2 (1:500; cat. no. GTX103322; Genetex, Inc.), NAD(P)H quinone dehydrogenase 1 (NQO1; 1:500; cat. no. GTX100235; Genetex, Inc.) and heme oxygenase 1 (HO-1; 1:500; cat. no. GTX101147; Genetex, Inc.). Blots were then incubated at $25^{\circ} \mathrm{C}$ for $1 \mathrm{~h}$ with a horseradish peroxidase-conjugated secondary antibody (1:1,000; cat. no. GB23302; Wuhan Servicebio Technology Co., Ltd.) and underwent chemiluminescence detection with the Molecular Imager ChemiDoc XRS system (Bio-Rad Laboratories, Inc.). Densitometric semi-quantification was performed using Image Lab 3.0 (Bio-Rad Laboratories, Inc.).

Statistical analysis. Data are presented as the mean \pm standard deviation. Results were analyzed by one-way ANOVA followed by Tukey's post hoc test using SPSS version 20 (IBM Corp.). $\mathrm{P}<0.05$ was considered to indicate a statistically significant difference.

\section{Results}

Effects of DMF on I/R-induced cellular damage. The cellular toxicity of different concentrations of DMF was assessed using the CCK- 8 assay (Fig. 2A). Treatment with $40 \mu \mathrm{M}$ DMF resulted in a significant decrease in cell viability; therefore, $20 \mu \mathrm{M}$ DMF was used for subsequent experimentation. Compared with the control group, I/R injury markedly decreased cell viability (Fig. 2B). Treatment with DMF significantly attenuated this effect in a dose-dependent manner, such that the viability of the DMF-treated cells was significantly higher than that of the I/R-treated cells. Furthermore, LDH release into the culture media was significantly elevated by $\mathrm{I} / \mathrm{R}$ induction (Fig. 2C) and was dose-dependently attenuated by DMF.

Effects of DMF on apoptosis. Compared with the control cells, a significant increase in Bax and a decrease in Bcl-2 were observed in I/R-treated cells, such that the apoptotic Bax/Bcl-2 index was significantly elevated (Fig. 3A). The expression levels of cleaved caspase-3 were also increased in 

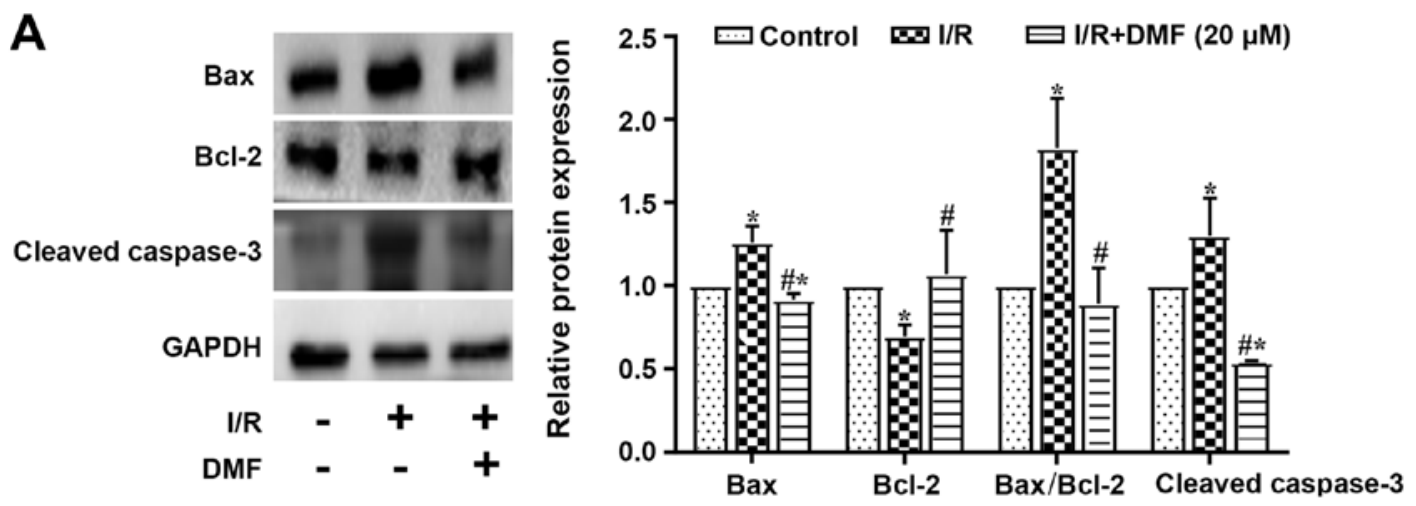

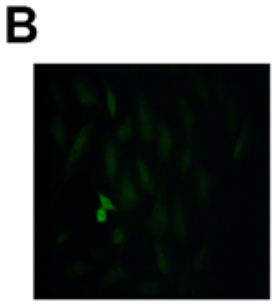

Control

C

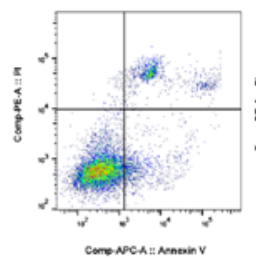

Control

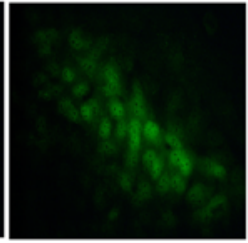

I/R

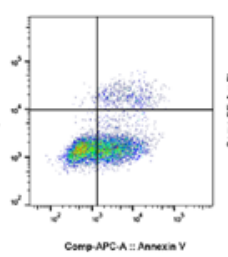

I/R

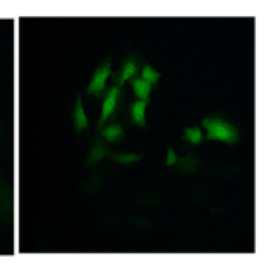

I/R+DMF (20 $\mu \mathrm{M})$

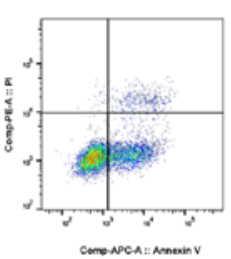

I/R+DMF $(20 \mu M)$
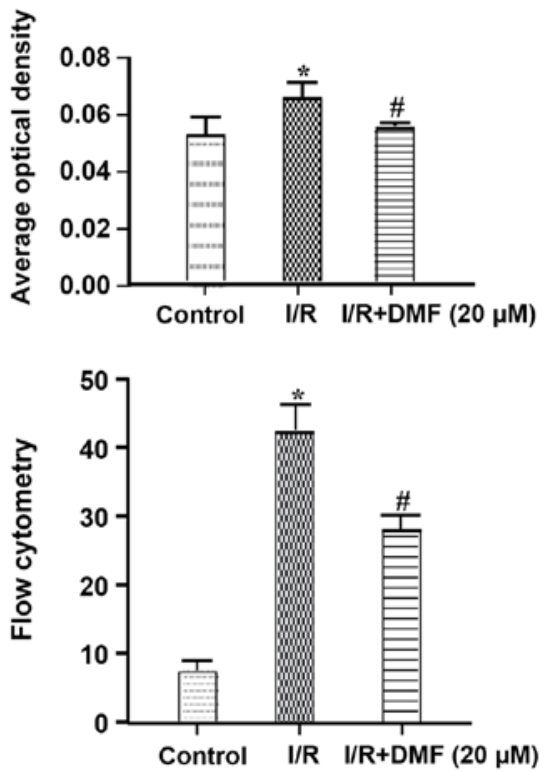

Figure 3. Effects of DMF $(20 \mu \mathrm{M})$ on apoptosis. (A) Relative expression levels of target proteins, as determined by western blotting in relation to the internal reference protein GAPDH. (B) TdT-mediated dUTP-biotin nick end labeling staining of apoptotic cells (magnification, $\mathrm{x} 200$ ) and densitometric semi-quantification. (C) Flow cytometric analysis. Cells double-stained with Annexin V and propidium iodide were differentiated into dead (Q1), late apoptotic (Q2), early apoptotic (Q3) and living cells (Q4). Quantification is shown in the right panel. ${ }^{*} \mathrm{P}<0.05$ vs. the control group; ${ }^{\#} \mathrm{P}<0.05$ vs. the $\mathrm{I} / \mathrm{R}$ group (n=3). Bcl-2, B-cell lymphoma 2; Bax, Bcl-2-associated X protein; I/R, ischemia/reperfusion; DMF, dimethyl fumarate; Q, quadrant.

the I/R-treated cells compared with those in the control group. The addition of DMF to the I/R-treated cells significantly decreased Bax, cleaved caspase-3 and the apoptotic index, and increased the expression levels of Bcl-2.

A greater number of TUNEL-positive cells was observed in the I/R-treated group than in the control group (Fig. 3B), and this was perturbed by treatment with $20 \mu \mathrm{M}$ DMF. The original flow cytometric data are depicted in Fig. 3C. The proportion of cells in quadrant 3 was significantly decreased in $\mathrm{I} / \mathrm{R}+\mathrm{DMF}$-treated cells compared with in the $\mathrm{I} / \mathrm{R}$ group.

Effects of DMF on ROS production. Compared with in the control cells, ROS levels in I/R-treated cells were increased, whereas DMF treatment attenuated this effect (Fig. 4A). Semi-quantitative analysis revealed that in comparison with the control group, the signal intensity of DCFH-DA staining was significantly amplified in the $\mathrm{I} / \mathrm{R}$ group $(\mathrm{P}<0.05$; Fig. $4 \mathrm{~B})$. The addition of DMF to the I/R-treated cells significantly reduced the signal intensity, which was lower than that in I/R-treated cells $(\mathrm{P}<0.05)$. Furthermore, the expression levels of HO-1 were lower in I/R-treated cells than in control cells $(\mathrm{P}<0.05)$, whereas DMF administration significantly increased
HO-1 expression (Fig. 4C). Similar results were obtained regarding NQO1 protein expression.

Effects of DMF on AKT/Nrf2 signaling. The ratio of p-AKT/AKT and the protein expression levels of Nrf2 are shown in Fig. 5. Compared with in the control group, I/R-treated cells exhibited a significantly decreased p-AKT/AKT ratio, which was markedly elevated above I/R levels by the addition of DMF. The expression levels of Nrf2 were decreased in I/R-treated cells compared with in the control group $(\mathrm{P}<0.05)$, but were significantly elevated by DMF administration $(\mathrm{P}<0.05)$.

\section{Discussion}

Coronary artery disease is characterized by myocardial ischemia and is currently the leading cause of death in the United States (25). Prolonged severe ischemia results in irreversible myocardial injury (26), which should be prevented where possible. Therefore, the potential for immediate/early interventions for myocardial reperfusion are important for the clinical outcome of ischemic heart disease (27). In previous 


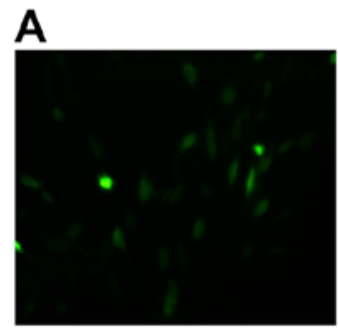

Control

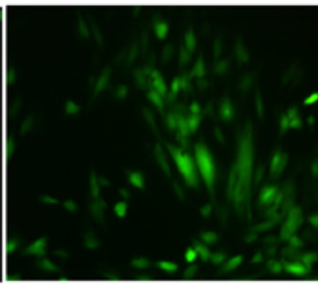

I/R

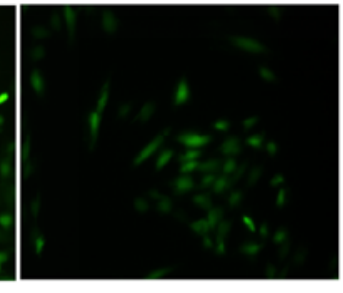

I/R+DMF (20 $\mu \mathrm{M})$
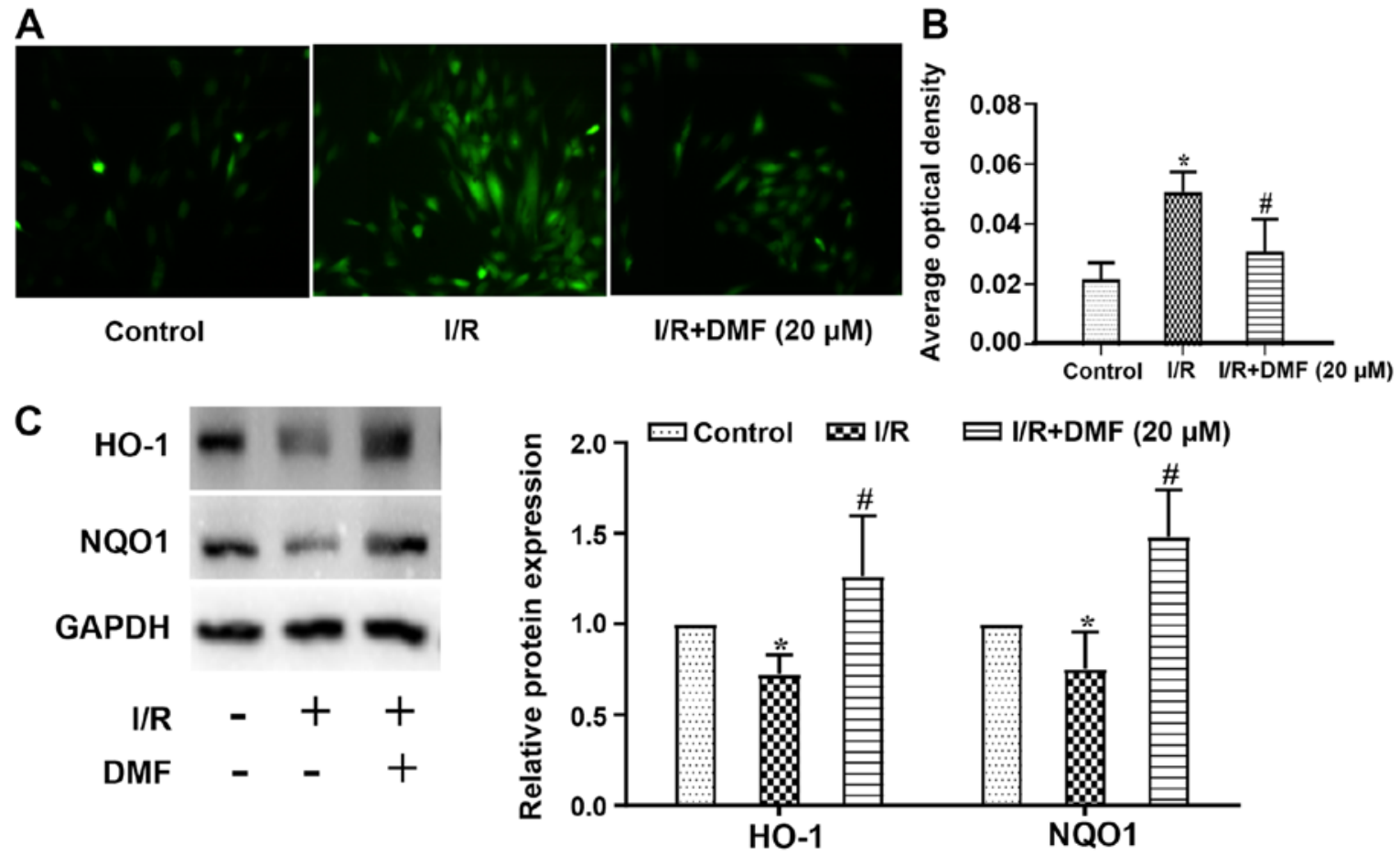

Figure 4. Effects of DMF $(20 \mu \mathrm{M})$ on oxidative stress. (A) Reactive oxygen species were detected by fluorescence to determine levels of oxidative stress (magnification, x200). (B) Semi-quantification of (A). (C) Expression levels of free radical scavengers HO-1 and NQO1 were determined by western blotting in relation to the internal reference protein GAPDH. ${ }^{*} \mathrm{P}<0.05$ vs. the control group; ${ }^{\#} \mathrm{P}<0.05$ vs. the $\mathrm{I} / \mathrm{R}$ group ( $\mathrm{n}=3$ ). I/R, ischemia/reperfusion; $\mathrm{DMF}$, dimethyl fumarate; HO-1, heme oxygenase 1; NQO1, NAD(P)H quinone dehydrogenase 1.

decades, advancements in timely reperfusion have been achieved $(5,26)$, which have significantly improved the clinical outcome of patients with ischemic heart disease (26). However, a number of studies have revealed that timely reperfusion of the myocardium may also bring about serious cardiac damage, termed myocardial I/R injury (4), which can at least partly undermine the beneficial effects of early reperfusion, and thus worsen clinical outcomes (28). Notably, timely reperfusion therapy itself has been reported as a cause of I/R, through occlusion of the coronary branch by balloon inflation and deflation during PCI, or aorta clamping and unclamping during CABG. Therefore, the ideal therapeutic strategy to manage ischemic heart disease would include both timely reperfusion and the prevention of I/R damage to the myocardium (29).

In the last decade, great improvements in timely perfusion have been achieved through PCI or CABG (2); however, treatments to prevent or reduce myocardial I/R injury are less than optimal (30). The results of several experimental studies appear promising $(31,32)$; however, such results are rarely translated into clinical studies; thus to date, there are a lack of established treatments to improve the clinical outcome of ischemic heart disease via the prevention or reduction of myocardial I/R injury $(4,26,30)$. In this context, the exploration of potential treatments to improve myocardial I/R injury is of great significance. The present study aimed to investigate the effects and underlying mechanisms of DMF on I/R damage in vitro.

Although the mechanisms responsible for I/R injury are not completely understood, a great deal of evidence has indicated that ROS production and apoptosis may have important roles in this event (33). DMF has historically been used to treat relapsing-remitting multiple sclerosis (34), and is currently regarded as an antioxidant that can modulate ROS production (35). Increasing evidence has suggested that DMF may have a profound impact on ROS production and apoptosis (36), and could exert protective effects against reperfusion injury to the liver (17) and brain (18). However, to the best of our knowledge, there are currently no studies reporting the effects of DMF on myocardial I/R injury.

The present study revealed that $\mathrm{I} / \mathrm{R}$ treatment significantly decreased cell viability and simultaneously increased LDH release into the culture media, a clear indication of cellular damage. These results are in accordance with previously reported findings $(37,38)$, which demonstrated the successful establishment of an I/R injury model. Notably, pretreatment with DMF significantly improved viability and reduced LDH release in I/R-treated cells in a dose-dependent manner, although I/R-associated damage could not be completely prevented. These findings suggested that DMF exerted distinct cellular protection against I/R injury.

The mechanisms underlying I/R-induced cellular injury have been extensively investigated (39). It is evident that apoptosis may serve a determining role in cellular damage, and augmented apoptotic activity is a well-known cause of I/R-associated myocardial injury $(40,41)$. In the present study, I/R clearly upregulated Bax and downregulated Bcl-2 expression, with a reflected increase in the apoptotic index (Bax/Bcl-2 ratio) and cleaved caspase-3 expression. Cleaved caspase- 3 plays an important role in apoptosis as it is the aggregation point of a number of apoptotic stimulating signaling pathways, such as the mitochondrial pathway, death receptor pathway and endoplasmic reticulum stress 

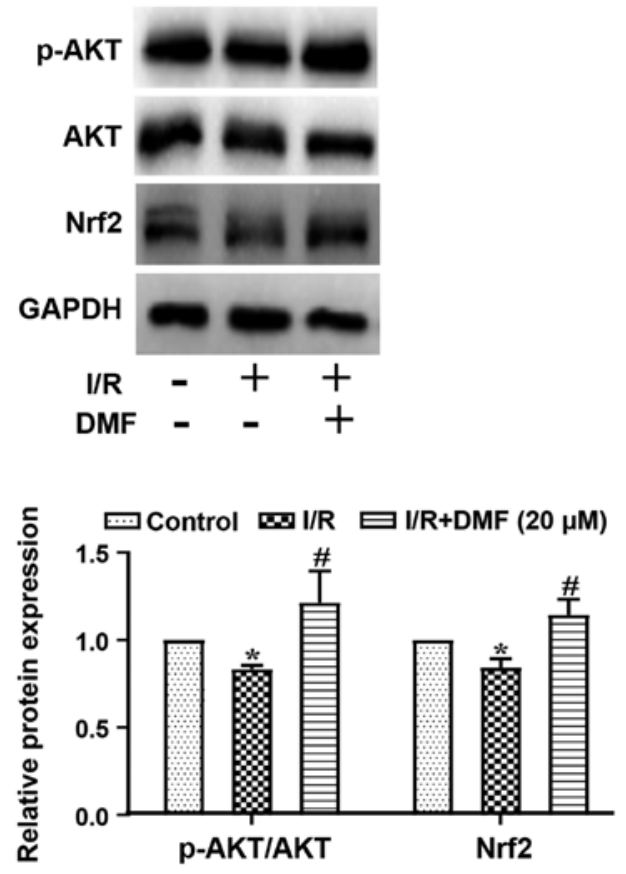

Figure 5. Effects of DMF $(20 \mu \mathrm{M})$ on the protein expression levels of AKT, $\mathrm{p}-\mathrm{AKT}$ and Nrf2, as determined by western blotting in relation to the internal reference protein GAPDH. "P $<0.05$ vs. the control group; ${ }^{\text {}} \mathrm{P}<0.05$ vs. the $\mathrm{I} / \mathrm{R}$ group $(\mathrm{n}=3)$. I/R, ischemia/reperfusion; DMF, dimethyl fumarate; Nrf2, nuclear factor erythroid 2-related factor 2; p-, phosphorylated.

pathway (42). Cleaved caspase-3 has been reported to break down various functional proteins to induce apoptosis; therefore, its activation is regarded as a sign of the irreversible stage of apoptosis after activation (43). The present study revealed that compared with in the control group, the expression levels of cleaved caspase-3 were increased in the I/R group and were decreased by $24-\mathrm{h}$ pretreatment with DMF; these results are consistent with those derived from TUNEL staining. The levels of these apoptotic indicators were comparable between the control and $\mathrm{I} / \mathrm{R}+\mathrm{DMF}$ groups, suggesting that DMF may have powerful anti-apoptotic effects. This was further supported by flow cytometric analysis, which indicated that the number of early apoptotic cells was significantly increased in the I/R-treated group compared with in the control group, whereas the proportion of living cells was notably decreased. DMF treatment also significantly decreased the number of early apoptotic cells and increased the proportion of living cells. Collectively, these results suggested that along with protection against I/R-induced cellular damage, DMF may significantly inhibit apoptotic activity, and that the protective effects of DMF against I/R-induced cellular damage could be associated with inhibition of apoptosis.

Although the factors that initiate apoptosis are not yet fully understood, a number of studies have shown that excessive ROS production may serve a critical role in the activation of apoptosis (44). ROS are hypothesized to mediate myocardial injury by inducing mitochondrial permeability transition pore expression (45) and dysfunction of the sarcoplasmic reticulum (46). In the present study, ROS production was significantly elevated by I/R-treatment, which, to a great extent, was counteracted by DMF, indicating that this treatment markedly suppressed ROS production. Furthermore,

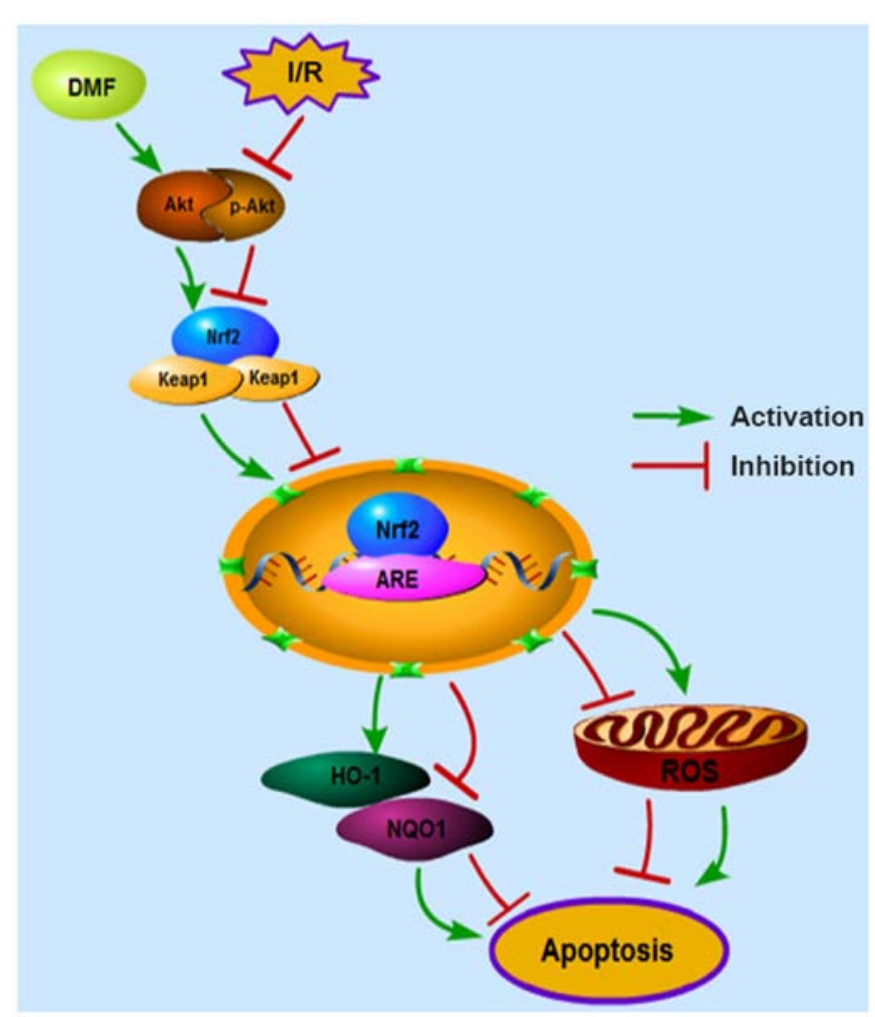

Figure 6. Mechanisms underlying the protective effects of DMF against myocardial I/R. I/R, ischemia/reperfusion; DMF, dimethyl fumarate; Nrf2, nuclear factor erythroid 2-related factor 2; p-, phosphorylated; Keap1, Kelch-like ECH-associated protein 1; ARE, antioxidant response elements; ROS, reactive oxygen species; HO-1, heme oxygenase 1; NQO1, NAD $(\mathrm{P}) \mathrm{H}$ quinone dehydrogenase 1 .

I/R significantly decreased the expression levels of the free radical scavengers HO-1 and NQO1, whereas their expression was completely restored by DMF. Therefore, on one hand, DMF may suppress ROS production, and on the other hand, it may augment the expression levels of free radical scavengers, which would beneficially modulate redox status (47) and further inhibit apoptosis (48). The results of the present study suggested that the protective effects of DMF on I/R-induced cellular damage may be associated with redox status modulation, which could lead to attenuation of apoptosis in response to $\mathrm{I} / \mathrm{R}$ insult.

It is well known that a series of pathways are involved in the regulation of redox status; among others, AKT and Nrf2 have been extensively investigated (49). In Nrf2-knockout mice, DMF failed to deliver protection against $I / R$ injury, which strongly indicated that DMF may act downstream of Nrf2 (18). Furthermore, previous studies have shown that Nrf2 can be activated via phosphorylation of AKT pathway components (50), which also upregulated HO-1 and NQO1 (51). In the present study, the p-AKT/AKT ratio was significantly decreased in I/R-treated cells, which was accompanied by a decrease in Nrf2 expression. Treatment with DMF markedly elevated the levels of p-AKT/AKT and Nrf2, indicating that DMF activity could be attributed to activation of the AKT/Nrf2 pathway.

Nrf2 is a putative transcription factor, and the suppression of Nrf2 activity is associated with an increase in Keap1, which sequesters Nrf2 in the cytoplasm and regulates its 
ubiquitin-dependent degradation (52). Upon activation by small molecules, the interaction between Keap1 and Nrf2 is disrupted. Nrf2 protein turnover is thereby attenuated, and the transcription factor translocates to the nucleus where it modulates transcription through AREs. Thus, a limitation of the present study is that it did not involve isolating nuclear proteins and comparing the expression of Nrf2 in the nuclear fraction of cells; this will be addressed in the future to support the data of the present study.

In conclusion, the myocardial protective functions of DMF were confirmed in a myocardial I/R model, and Nrf2 modulation was validated as a primary mechanism for inhibiting oxidative stress and apoptosis (Fig. 6). Currently, DMF has been confirmed to suppress cardiovascular diseases, such as pulmonary hypertension and diabetic cardiomyopathy. Based on the present findings, modulation of the AKT/Nrf2 pathway by DMF may be a promising treatment option for patients with acute ischemic heart disease. With continuous in-depth research on $\mathrm{DMF}$, it is expected to become a future treatment for myocardial I/R injury and pave the way for improved clinical applications.

\section{Acknowledgements}

Not applicable.

\section{Funding}

The present study was supported by grants from the National Natural Science Foundation of China (grant nos. 81370250 and 81974026) and the Hunan Provincial Natural Science Foundation of China (grant no. 2017SK50108).

\section{Availability of data and materials}

All data generated or analyzed during this study are included in this published article.

\section{Authors' contributions}

QM conceived the study and designed the experiments. YK, YZ and ZX performed the experiments. LX and PW analyzed the data and drafted the manuscript. All authors read and approved the final manuscript.

\section{Ethics approval and consent to participate}

Not applicable.

\section{Patient consent for publication}

Not applicable.

\section{Competing interests}

The authors declare that they have no competing interests.

\section{References}

1. Yoon J, Seo H, Oh IH and Yoon SJ: The non-communicable disease burden in Korea: Findings from the 2012 Korean burden of disease study. J Korean Med Sci 31 (Suppl 2): S158-S167, 2016.
2. Yetgin T, Manintveld OC, Boersma E, Kappetein AP, van Geuns RJ, Zijlstra F, Duncker DJ and van der Giessen WJ: Remote ischemic conditioning in percutaneous coronary intervention and coronary artery bypass grafting. Circ J 76: 2392-2404, 2012.

3. Bompotis GC, Deftereos S, Angelidis C, Choidis E, Panagopoulou V, Kaoukis A, Vassilikos VP, Cleman MW and Giannopoulos G: Altered calcium handling in reperfusion injury. Med Chem 12: 114-130, 2016.

4. Ibáñez B, Heusch G, Ovize M and Van de Werf F: Evolving therapies for myocardial ischemia/reperfusion injury. J Am Coll Cardiol 65: 1454-1471, 2015.

5. Binder A, Ali A, Chawla R, Aziz HA, Abbate A and Jovin IS Myocardial protection from ischemia-reperfusion injury post coronary revascularization. Expert Rev Cardiovasc Ther 13: 1045-1057, 2015

6. Kern KB, Hanna JM, Young HN, Ellingson CJ, White JJ, Heller B, Illindala U, Hsu CH and Zuercher M: Importance of both early reperfusion and therapeutic hypothermia in limiting myocardial infarct size post-cardiac arrest in a porcine model. JACC Cardiovasc Interv 9: 2403-2412, 2016.

7. Wang S, Zhang F, Zhao G, Cheng Y, Wu T, Wu B and Zhang YE: Mitochondrial PKC- $\varepsilon$ deficiency promotes I/R-mediated myocardial injury via GSK3 $\beta$-dependent mitochondrial permeability transition pore opening. J Cell Mol Med 21: 2009-2021, 2017.

8. Neri M, Riezzo I, Pascale N, Pomara C and Turillazzi E: Ischemia/reperfusion injury following acute myocardial infarction: A critical issue for clinicians and forensic pathologists. Mediators Inflamm 2017: 7018393, 2017.

9. Granger DN and Kvietys PR: Reperfusion injury and reactive oxygen species: The evolution of a concept. Redox Biol 6: 524-551, 2015.

10. Sanderson TH, Reynolds CA, Kumar R, Przyklenk K and Hüttemann M: Molecular mechanisms of ischemia-reperfusion injury in brain: Pivotal role of the mitochondrial membrane potential in reactive oxygen species generation. Mol Neurobiol 47: 9-23, 2013.

11. Sifuentes-Franco S,Pacheco-Moisés FP, Rodríguez-Carrizalez AD and Miranda-Díaz AG: The role of oxidative stress, mitochondrial function, and autophagy in diabetic polyneuropathy. J Diabetes Res 2017: 1673081,2017.

12. Bhat AH, Dar KB, Anees S, Zargar MA, Masood A, Sofi MA and Ganie SA: Oxidative stress, mitochondrial dysfunction and neurodegenerative diseases; a mechanistic insight. Biomed Pharmacother 74: 101-110, 2015.

13. Sinha K, Das J, Pal PB and Sil PC: Oxidative stress: The mitochondria-dependent and mitochondria-independent pathways of apoptosis. Arch Toxicol 87: 1157-1180, 2013.

14. Boshra V and Atwa A: Effect of cerebrolysin on oxidative stress-induced apoptosis in an experimental rat model of myocardial ischemia. Physiol Int 103: 310-320, 2016.

15. Herr DJ, Aune SE and Menick DR: Induction and assessment of ischemia-reperfusion injury in Langendorff-perfused rat hearts. J Vis Exp 101: e52908, 2015.

16. Heusch G and Gersh BJ: The pathophysiology of acute myocardial infarction and strategies of protection beyond reperfusion: A continual challenge. Eur Heart J 38: 774-784, 2017.

17. Takasu C, Vaziri ND, Li S, Robles L, Vo K, Takasu M, Pham C, Farzaneh SH, Shimada M, Stamos MJ, et al: Treatment with dimethyl fumarate ameliorates liver ischemia/reperfusion injury. World J Gastroenterol 23: 4508-4516, 2017.

18. Yao Y, Miao W, Liu Z, Han W, Shi K, Shen Y, Li H, Liu Q, Fu Y, Huang D, et al: Dimethyl fumarate and monomethyl fumarate promote post-ischemic recovery in mice. Transl Stroke Res 7: 535-547, 2016.

19. Li J, Ma J, Lacagnina MJ, Lorca S, Odem MA, Walters ET, Kavelaars A and Grace PM: Oral dimethyl fumarate reduces peripheral neuropathic pain in rodents via NFE2L2 antioxidant signaling. Anesthesiology, 132: 343-356, 2020.

20. Ghadiri M, Rezk A,Li R, Evans A,Luessi F,Zipp F, Giacomini PS, Antel J and Bar-Or A: Dimethyl fumarate-induced lymphopenia in MS due to differential T-cell subset apoptosis. Neurol Neuroimmunol Neuroinflamm 4: e340, 2017.

21. Belcher JD, Chen C, Nguyen J, Zhang P, Abdulla F, Nguyen P, Killeen T, Xu P, O'Sullivan G, Nath KA, et al: Control of Oxidative Stress and Inflammation in Sickle Cell Disease with the Nrf2 Activator Dimethyl Fumarate. Antioxid Redox Signal 26: 748-762, 2017.

22. Sghaier R, Nury T, Leoni V, Caccia C, Pais De Barros JP, Cherif A, Vejux A, Moreau T, Limem K, et al: Dimethyl fumarate and monomethyl fumarate attenuate oxidative stress and mitochondrial alterations leading to oxiapoptophagy in $158 \mathrm{~N}$ murine oligodendrocytes treated with 7 $\beta$-hydroxycholesterol. J Steroid Biochem Mol Biol 194: 105432, 2019. 
23. Crowley LC, Marfell BJ, Scott AP and Waterhouse NJ: Quantitation of apoptosis and necrosis by Annexin V binding, propidium iodide uptake, and flow cytometry. Cold Spring Harb Protoc 2016: 953-957, 2016

24. Koç E, Çelik-Uzuner S, Uzuner U and Çakmak R: The detailed comparison of cell death detected by Annexin V-PI counterstain using fluorescence microscope, flow cytometry and automated cell counter in mammalian and microalgae cells. J Fluoresc 28 : $1393-1404,2018$

25. Dalen JE, Alpert JS, Goldberg RJ and Weinstein RS: The epidemic of the 20(th) century: Coronary heart disease. Am J Med 127: 807-812, 2014.

26. Chi HJ, Chen ML, Yang XC, Lin XM, Sun H, Zhao WS, Qi D, Dong JL and Cai J: Progress in therapies for myocardial ischemia reperfusion injury. Curr Drug Targets 18: 1712-1721, 2017.

27. Kleinbongard P, Skyschally A and Heusch G: Erratum to: Cardioprotection by remote ischemic conditioning and its signal transduction. Pflugers Arch 469: 843, 2017.

28. Heusch G: Molecular basis of cardioprotection: Signal transduction in ischemic pre-, post-, and remote conditioning. Circ Res 116: 674-699, 2015.

29. Bernink FJ, Timmers L, Beek AM, Diamant M, Roos ST, Van Rossum AC and Appelman Y: Progression in attenuating myocardial reperfusion injury: An overview. Int J Cardiol 170: 261-269, 2014.

30. Hausenloy DJ and Yellon DM: Myocardial ischemia-reperfusion injury: A neglected therapeutic target. J Clin Invest 123: 92-100, 2013.

31. Ndrepepa G, Colleran R and Kastrati A: Reperfusion injury in ST-segment elevation myocardial infarction: The final frontier. Coron Artery Dis 28: 253-262, 2017.

32. Frank A, Bonney M, Bonney S, Weitzel L, Koeppen M and Eckle T: Myocardial ischemia reperfusion injury: From basic science to clinical bedside. Semin Cardiothorac Vasc Anesth 16 123-132, 2012.

33. Guo W, Liu X, Li J, Shen Y, Zhou Z, Wang M, Xie Y, Feng X, Wang $\mathrm{L}$ and $\mathrm{Wu} \mathrm{X}$ : Prdx 1 alleviates cardiomyocyte apoptosis through ROS-activated MAPK pathway during myocardial ischemia/reperfusion injury. Int J Biol Macromol 112: 608-615, 2018.

34. Huisman E, Papadimitropoulou K, Jarrett J, Bending M, Firth Z, Allen $\mathrm{F}$ and Adlard N: Systematic literature review and network meta-analysis in highly active relapsing-remitting multiple sclerosis and rapidly evolving severe multiple sclerosis. BMJ Open 7: e013430, 2017.

35. Akino N, Wada-Hiraike O, Terao $\mathrm{H}$, Honjoh $\mathrm{H}$, Isono $\mathrm{W}, \mathrm{Fu} \mathrm{H}$ Hirano M, Miyamoto Y, Tanikawa M, Harada M, et al: Activation of Nrf2 might reduce oxidative stress in human granulosa cells. Mol Cell Endocrinol 470: 96-104, 2018.

36. Ohl K, Tenbrock K and Kipp M: Oxidative stress in multiple sclerosis: Central and peripheral mode of action. Exp Neurol 277 58-67, 2016.

37. Chen Y, Wang H, Zhang Y, Wang Z, Liu S and Cui L: Pretreatment of ghrelin protects H9c2 cells against hypoxia/reoxygenation-induced cell death via PI3K/AKT and AMPK pathways. Artif Cells Nanomed Biotechnol 47: 2179-2187, 2019.

38. Fan L, Zhou W, Zhang L, Jiang D, Zhao Q and Liu L: Sitagliptin protects against hypoxia/reoxygenation (H/R)-induced cardiac microvascular endothelial cell injury. Am J Transl Res 11: 2099-2107, 2019
39. Moe GW and Marín-García J: Role of cell death in the progression of heart failure. Heart Fail Rev 21: 157-167, 2016.

40. Qian W, Wang Z, Xu T and Li D: Anti-apoptotic effects an mechanisms of salvianolic acid A on cardiomyocytes in ischemia-reperfusion injury. Histol Histopathol 34: 223-231, 2019.

41. Lejay A, Fang F, John R, Van JA, Barr M, Thaveau F, Chakfe N, Geny B and Scholey JW: Ischemia reperfusion injury, ischemic conditioning and diabetes mellitus. J Mol Cell Cardiol 91: 11-22, 2016.

42. Chen Y, Feng X, Hu X, Sha J, Li B, Zhang H and Fan H: Dexmedetomidine ameliorates acute stress-induced kidney injury by attenuating oxidative stress and apoptosis through inhibition of the ROS/JNK signaling pathway. Oxid Med Cell Longev 2018: 4035310, 2018.

43. Crowley LC and Waterhouse NJ: Detecting cleaved caspase-3 in apoptotic cells by flow cytometry. Cold Spring Harb Protoc: Nov 1, 2016 (Epub ahead of print).

44. Liu YF, Chu YY, Zhang XZ,Zhang M, Xie FG, Zhou M, Wen HH and Shu AH: TGF $\beta 1$ protects myocardium from apoptosis and oxidative damage after ischemia reperfusion. Eur Rev Med Pharmacol Sci 21: 1551-1558, 2017.

45. Cadenas S: ROS and redox signaling in myocardial ischemia-reperfusion injury and cardioprotection. Free Radic Biol Med 117: 76-89, 2018.

46. Hall AR, Burke N, Dongworth RK, Kalkhoran SB, Dyson A, Vicencio JM, Dorn GW II, Yellon DM and Hausenloy DJ: Hearts deficient in both Mfn1 and Mfn2 are protected against acute myocardial infarction. Cell Death Dis 7: e2238, 2016.

47. Carlström KE, Ewing E, Granqvist M, Gyllenberg A, Aeinehband S, Enoksson SL, Checa A, Badam TVS, Huang J, Gomez-Cabrero D, et al: Therapeutic efficacy of dimethyl fumarate in relapsing-remitting multiple sclerosis associates with ROS pathway in monocytes. Nat Commun 10: 3081, 2019.

48. Han G and Zhou Q: Dimethylfumarate induces cell cycle arrest and apoptosis via regulating intracellular redox systems in HeLa cells. In Vitro Cell Dev Biol Anim 52: 1034-1041, 2016.

49. Hu YR, Ma H, Zou ZY, He K, Xiao YB, Wang Y, Feng M, Ye XL and Li XG: Activation of Akt and JNK/Nrf2/NQO1 pathway contributes to the protective effect of coptisine against AAPH-induced oxidative stress. Biomed Pharmacother 85: 313-322, 2017.

50. Ci X, Zhou J, Lv H, Yu Q, Peng L and Hua S: Betulin exhibits anti-inflammatory activity in LPS-stimulated macrophages and endotoxin-shocked mice through an AMPK/AKT/Nrf2-dependent mechanism. Cell Death Dis 18: 8: e2798, 2017.

51. Wu PS, Ding HY, Yen JH, Chen SF, Lee KH and Wu MJ: Anti-inflammatory activity of 8 -hydroxydaidzein in LPS-stimulated BV2 microglial cells via activation of Nrf2-antioxidant and attenuation of Akt/NF- $\kappa \mathrm{B}$-inflammatory signaling pathways, as well as inhibition of COX-2 activity. J Agric Food Chem 66: 5790-5801, 2018.

52. Bellezza I, Giambanco I, Minelli A and Donato R: Nrf2-Keap1 signaling in oxidative and reductive stress. Biochim Biophys Acta Mol Cell Res 1865: 721-733, 2018.

(2) This work is licensed under a Creative Commons

C ${ }_{\mathrm{EY}}$ No ND Attribution-NonCommercial-NoDerivatives 4.0 International (CC BY-NC-ND 4.0) License. 